\title{
The Liver Protection Effect of Different Polarity Fractions of Semen Astragali Complanati Wine on Mice
}

\author{
Xuehui Fan ${ }^{1,2,}$, Qingan Zhang ${ }^{2}$, Chengrui Tian² \\ ${ }^{1}$ School of Physical Education, Shaanxi Normal University, Xi'an, Shaanxi Province, China. \\ ${ }^{2}$ College of Food Engineering and Nutritional Science, Shaanxi Normal University, Xi'an, Shaanxi Province, \\ China. \\ afanxuehui012008@snnu.edu.cn
}

Keywords: semen astragali complanati wine; mice; in vivo; liver

\begin{abstract}
In this paper, healthy mice are exposed to different polarity extracts of semen astragali complanati wine, ether, ethyl acetate and water through intragastric administration for 30 days. Then the contents of superoxide dismutase (SOD), malonaldehyde (MDA), catalase (CAT), total antioxidant capacity (T-AOC), glutathione (GSH), glutathione peroxidase (GSH-PX) and protein carbonyl in rats' serum are detected, as well as the polyphone contents of different polarity extracts. The results show that semen astragali complanati wine and its different polarity extracts increase the antioxidant capacity of rats' liver. Rats from water extracts group have more polyphenol; but their antioxidant capacities are worse than rats from ether group and ethyl acetate group. Rats exposed to semen astragali complanati wine have the strongest antioxidant abilities. It indicates that compared with the low polarity extracts of ether and ethyl acetate, as well as the high polarity extract of water, diversified polarity extracts in the astragali complanati wine can produce synergistic effect, and help rats acquire better antioxidant capacity.
\end{abstract}

\section{Introduction}

Semen Astragali Complanati means the dry, ripe seeds of Astragali complanati R. Br., a kind of perennial dicotyledonous plant. Its features are recorded in the Compendium of Materia Medica as warm in nature, sweet taste, non-toxic, and attributable to liver and kidney channels. According to the book, semen astragali complanati can nourish liver and kidney, cure kidney disease, and benefit to liver and eyesight. It is a traditional Chinese medicine which can tonify liver and kidney. Modern medical literature reports, flavone parts of semen astragali complanati can significantly reduce triglyceride in blood, and has hepatoprotective and lipid-lowering effects. [1] Semen astragali complanati extracts have strong protective effects against liver damage; the effects are more obvious in water solution parts; the mechanism may be related to the inhibition of lipid peroxidation. Semen astragali complanati flavone is one of the main effective components [2]. The preventive administration of flavones of semen astragali complanati can inhibit the formation of liver fibrosis induced by DMN in rats, which may be related to the regulatory cytokine IFN $\gamma$ and TGF $\beta 1$ [3]. Flavone of semen astragali complanati can significantly improve the activity of liver cells, promote the proliferation of hepatocytes and significantly reduce the degree of liver tissue damage; it has significant effects in liver protection [4]. The effects of semen astragali complanati are related to the improvement of liver and kidney functions; phenols and flavonoid composition of semen astragali complanati are physiological activity [5-6]; the mechanism may be related to the inhibition of lipid peroxidation.

In this experiment, the direct fermentation method which uses yeast strain is adopted to brew semen astragali complanati wine for the first time. The liquid fermentation process is employed together with dregs fermentation method which combines the advantages of liquid fermentation and solid state fermentation. Compared with existing processing methods of hot salt frying, baking with honey, smoking, steaming with wine and boiling, this fermentation method is carried out under 
normal temperature and pressure conditions; it can preserve active ingredients of semen astragali complanati. In the biological transformation process, clastic enzyme of microorganism dissolves cell walls, then the cells can leach more effective components; big molecules can be degraded into small molecules which are easily absorbed by human body. Passing through blood-brain barrier, these small molecules play their roles by combining with human body cell protein. In order to analyze the effective components of astragali complanati wine in liver protection, this paper evaluates the antioxidant activities of different polarity polyphenol extracts and their mechanisms, hoping to provide theoretical basis for further research and development of pharmacology.

\section{Experiment Materials and Apparatus}

\subsection{Experiment animal and feed}

Experiment animals are healthy, Kunming ICR male mice, body mass $(24+3) \mathrm{g}$. Animal food basically comes from the Experimental Animal Center of Xi'an Jiaotong University.

\subsection{Reagents}

Reagents include semen astragali complanati (purchased from Northwestern Medicine Market, Xi'an, Shaanxi Province), Angel yeast (produced by Angel Yeast Co., Ltd, Yichang, Hubei Province), semen astragali complanati wine (made by Food Processing and Quality Control Laboratory), rutin (produced by Xi'an KinGreen Bio-engineering Technology co. LTD), superoxide dismutase (SOD) test box and other test boxes (produced by Nanjing Jiancheng Bioengineering Institute).

\subsection{Apparatus}

Apparatus used in this experiment include AB204-N electronic analytical balance (produced by Mettler Toledo Instruments (Shanghai) Co., Ltd), Multiskan Go ELIASA (produced by Thermo Scientific), TU-1810 UV VIS spectrophotometer (produced by Beijing Persee General Instrument Co., Ltd.) and TGL-16B desk centrifuge (produced by Shanghai Anting Scientific Instrument Factory).

\section{Research Method}

\subsection{The brewing process of semen astragali complanati wine}

Selecting seeds $\rightarrow$ cleaning $\rightarrow$ draining off $\rightarrow$ water immersion and germination $\rightarrow$ drying $\rightarrow$ baking $\rightarrow$ crushing $\rightarrow$ sugar adjusting

\section{NaHSO3 inoculation}

Acidity adjusting $\rightarrow$ sterilization $\rightarrow$ main fermentation (sugar adjusting for 1 time) $\rightarrow$ filtration $\rightarrow$ aging $\rightarrow$ clarification $\rightarrow$ freezing $\rightarrow$ bottling $\rightarrow$ finished products

The optimum fermentation conditions are 26 degree, $3.7 \mathrm{pH}$ value, $1.1 \%$ yeast addition and $87 \mathrm{ppm}$ SO2 addition.

\subsection{Production of different polarity extracts of semen astragali complanati wine}

$50 \mathrm{ml}$ aging astragali complanati wine is added with ether according to the volume ratio of 1:1. After shaking and letting stand until the solution is layered, the upper part of ether extraction is retained. After continuous extraction for 3 times, putting all extractions together, then XAD-7 macroporous resin is used to remove protein and sugar in the solution. Then the solution is put into vacuum rotary evaporation instrument at 40 degree for vacuum concentration to recycle petroleum ether. Ether extract concentrated liquid is obtained. Using $7.4 \%$ ethanol to dissolve the constant volume of $50 \mathrm{ml}$ in a brown flask, the same volume petroleum ether extract is obtained. The remaining solution is processed according to above steps with $50 \mathrm{ml}$ ethyl acetate extraction for 3 times; macroporous resin is used to remove protein and sugar in the solution. After rotary evaporation, the concentrated liquid of ethyl acetate extract is obtained. Using $7.4 \%$ ethanol to dissolve in constant 
volume of $50 \mathrm{ml}$, the ethyl acetate extract is obtained. The remaining extraction is dissolved with $7.4 \%$ ethanol solution in constant volume of $50 \mathrm{ml}$; the water extract of semen astragali complanati wine is obtained. The extracts are stored in the refrigerator for following experiment.

\subsection{Administration}

Rats with similar weights are randomly divided into 5 groups; each group has 8 rats. The five groups are blank control group (distilled water $0.01 \mathrm{~mL} /(\mathrm{g} \mathrm{bw} \cdot \mathrm{d})$, ether group $(0.01 \mathrm{~mL} /(\mathrm{g} \mathrm{bw} \cdot \mathrm{d})$ ), ethyl acetate group $(0.01 \mathrm{~mL} /(\mathrm{g} \mathrm{bw} \cdot \mathrm{d}))$, water extract group $(0.01 \mathrm{~mL} /(\mathrm{g} \mathrm{bw} \cdot \mathrm{d}))$ and semen astragali complanati wine group $(0.01 \mathrm{~mL} /(\mathrm{g} b \mathrm{~b} \cdot \mathrm{d}))$. One time per day intragastric administration starts after 7 days adaptation feeding, and lasts for 30 days. The daily consumption of food is recorded; the weights of rats are recorded every 7 days.

\subsection{Animal execution and index detection method}

After 30 days' intragastric administration, water and food are no longer provided. The rats are killed through cervical vertebra dislocation method at the thirty-first day. Rats' livers and kidneys are weighed and recorded.

Liver homogenate is prepared as following steps: $0.5000 \mathrm{~g}$ liver is smashed in $4.5 \mathrm{ml}$ normal saline (0.9\%); then the homogenate is centrifuged for 10 minus at the speed of $3500 \mathrm{r} / \mathrm{min}$. The supernatant liquor is taken and conserved to be detected. The T-AOC, SOD, CAT, GSH-Px activity, protein carbonyl content, MDA content and GSH content of liver homogenate are measured according to instructions of test boxes.

\subsection{Determination of total flavonoid contents $[8-9,11]$}

Rutin is used as the standard sample for determination. $1 \mathrm{ml}$ sample liquid is taken out and added with $0.3 \mathrm{ml} \mathrm{NaNO2}$ solution. After reaction for 6 minutes, another $0.3 \mathrm{ml}$ is added. After 6 minutes' reaction with $10 \% \mathrm{Al}(\mathrm{NO} 3) 3,4 \mathrm{ml} 4 \% \mathrm{NaOH}$ and $10 \mathrm{ml} \mathrm{30 \%} \mathrm{ethanol} \mathrm{are} \mathrm{added} \mathrm{and} \mathrm{shaken.} \mathrm{After}$ coloration for $15 \mathrm{mins}$, the sample is measured at $510 \mathrm{~nm}$. The regression equation of the concentration of rutin solution $(\mathrm{g} / \mathrm{ml})$ and the absorbance, A is gotten: $y=0.0008 \mathrm{x}-0.0089, \mathrm{R} 2$ $=0.9974$ (in which y means absorbance, $\mathrm{X}$ means rutin concentration). Determining the absorbance of A at $510 \mathrm{~nm}$ in accordance with above method, then the concentration of flavonoid is obtained according to the standard curve regression.

\subsection{Data processing}

The data are analyzed by SPSS 19.0 statistical software. All data are represented as $(\mathrm{x} \pm \mathrm{s})$. The significant differences between groups are analyzed through one-way analysis of variance and the SSR method. $\mathrm{P}<0.05$ indicates statistically significant difference.

\section{Experiment Results}

\subsection{The effects of semen astragali complanati wine and its different polar extracts on the weights of rats}

After 30 days' gavage, compared with the blank control group, the growth, mental status, diet and body weight conditions of rats from the four groups remain normal. At the thirtieth day of the experiment, the average weight increases of rats in the semen astragali complanati wine group, the water extract group, the ethyl acetate group and the ether group are $11.65 \%, 14.48 \%, 23.20 \%$, and $26.20 \%$. Compared with rats in the blank control group (28.33\%), figures of semen astragali complanati wine group and water extract group decrease significantly. It can be seen that different extracts of semen astragali complanati have inhibitory effects on the weight growth of mice. 


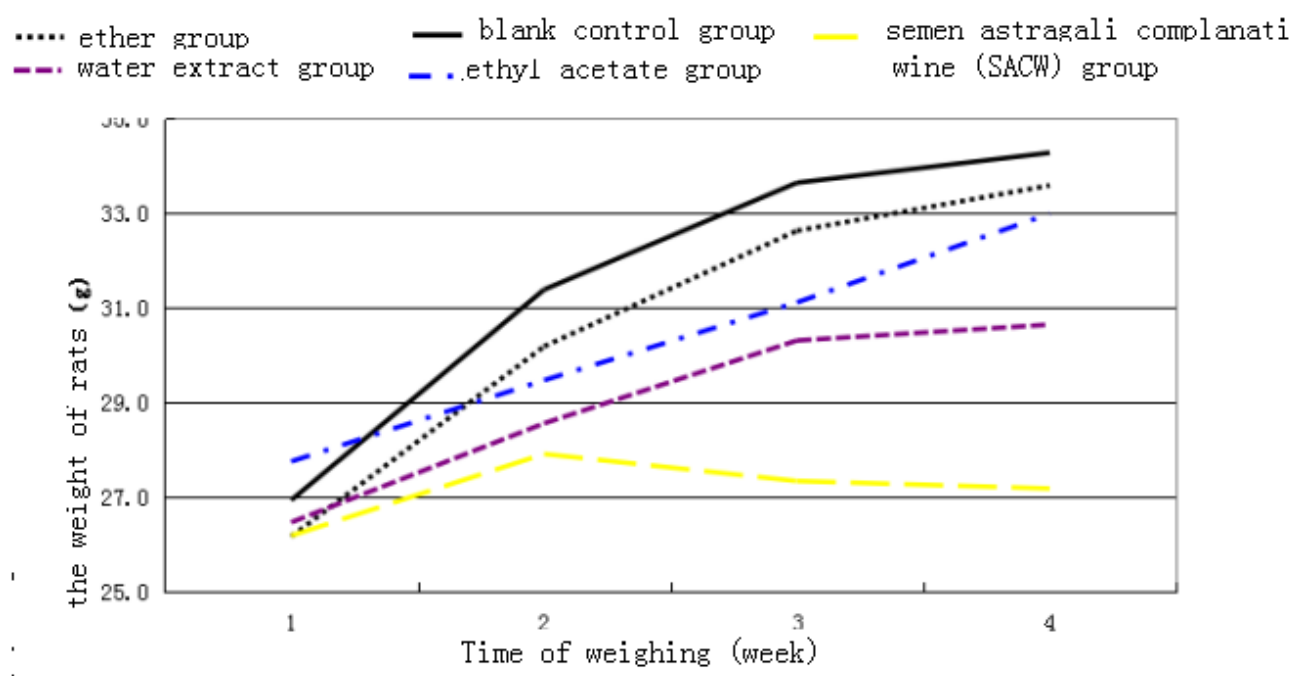

Figure .1 Effects of SACW on the increase of rats' body weight

\subsection{The effects of semen astragali complanati wine and its different polarity extracts on viscera indexes of rats}

As can be seen from Table 1, semen astragali complanati wine and its different polarity extracts have no significant influences on the heart index of mice. Compared with the control group, spleen indexes of rats from SACW group and ether group slightly increase. No significant difference is found in the spleen indexes of rats from the ethyl acetate group and water extract group. It can be concluded that semen astragali complanati wine and its various polarity extracts do not damage the organs of mice, and do not have toxic or side effects.

Table 1. Effects of SACW on visceral index (\%)

\begin{tabular}{lll}
\hline Group & Liver index $(\%)$ & $\begin{array}{l}\text { spleen index } \\
(\%)\end{array}$ \\
\hline blank control group & $45.12 \pm 4.10$ & $3.74 \pm 0.33$ \\
ether group & $44.91 \pm 5.73$ & $3.95 \pm 0.26$ \\
ethyl acetate group & $44.62 \pm 1.28$ & $3.61 \pm 0.15$ \\
water extract group & $45.22 \pm 2.94$ & $3.67 \pm 0.18$ \\
SACW group & $45.31 \pm 1.01$ & $4.26 \pm 0.05$ \\
\hline
\end{tabular}

Note: different letters indicate the significant differences between different groups (columns). The lowercase letters show significant differences $(\mathrm{P}<0.05)$, while the upper case letters indicate extremely significant differences $(\mathrm{p}<0.01)$; similarly hereinafter.

\subsection{Effects of semen astragali complanati wine and its different polarity extracts on GSH, protein carbonyl, MDA, T-AOC, SOD, CAT and GSH-Px in rats' liver tissue}

From Table 2 and Table 3 it can be found, for rats from the ether group, their GSH, T-AOC, SOD contents and GSH-Px activity of livers significantly increase $(\mathrm{p}<0.05)$; their protein carboxyl and MDA contents significantly decrease $(\mathrm{p}<0.01)$. For the ethyl acetate group, their contents of $G S H$, T-AOC, SOD and CAT in liver tissue significantly increase $(\mathrm{p}<0.05)$; their content of protein carboxyl significantly decreases $(\mathrm{p}<0.05)$; their MDA content extremely significantly decreases $(p<0.01)$; their GSH-Px activity extremely significantly increases $(p<0.01)$. For the water extract group, the content of MDA in liver tissue significantly decreases $(\mathrm{p}<0.05)$; the activities of T-AOC, SOD and GSH-Px significantly increase $(\mathrm{p}<0.05)$; the content of GSH significantly increases $(p<0.05)$. Above results indicate that semen astragali complanati wine extracts can decrease in vivo oxidative damage of proteins, increase oxidase system activity, protect liver and improve body antioxidant capacity. It can be concluded that semen astragali complanati wine has the function of liver protection. 
Table 2. Effects of SACW and its different polar extractions on T-AOC, SOD activity, protein carbonyl and MDA contents of rats' liver tissue

\begin{tabular}{|c|c|c|c|c|c|}
\hline group & $\mathrm{n}$ & $\begin{array}{l}\text { protein carbonyl } \\
\text { (nmol/mg prot) }\end{array}$ & $\begin{array}{l}\text { T-AOC } \\
\text { (U/mg prot) }\end{array}$ & $\begin{array}{l}\text { SOD } \\
\text { (U/mg prot) }\end{array}$ & $\begin{array}{l}\text { MDA } \\
\text { (nmol/mg prot) }\end{array}$ \\
\hline \multicolumn{6}{|l|}{ blank control } \\
\hline $\begin{array}{l}\text { group } \\
\text { ether group }\end{array}$ & 8 & $56.39 \pm 5.94$ & $\begin{array}{l}36.80 \pm 6.74 \\
73.77+53^{a}\end{array}$ & $629.09 \pm 43.78$ & $\begin{array}{l}4.77 \pm 0.22 \\
1.95+021^{a}\end{array}$ \\
\hline $\begin{array}{l}\text { ether group } \\
\text { ethyl acetate }\end{array}$ & 8 & $42.23 \pm 4.39^{\circ}$ & $73.97 \pm 5.73$ & $828.42 \pm 81.66$ & $1.95 \pm 0.21$ \\
\hline group & 8 & $39.04 \pm 2.99^{\mathrm{a}}$ & $69.82 \pm 11.70^{\mathrm{a}}$ & $793.98 \pm 68.87^{\mathrm{a}}$ & $3.32 \pm 0.15^{\mathrm{ab}}$ \\
\hline group & 8 & $55.32 \pm 5.81^{\text {bc }}$ & $51.24 \pm 11.18^{\mathbf{a b}}$ & $724.69 \pm 91.18^{\mathbf{a b}}$ & $3.35 \pm 0.09^{\mathbf{a b}}$ \\
\hline SACW group & 8 & $23.36 \pm 1.07^{\mathbf{A D C D}}$ & $81.43 \pm 5.81^{\text {Abca }}$ & $915.08 \pm 45.44^{\text {Aca }}$ & $1.09 \pm 0.11^{\mathrm{AbCD}}$ \\
\hline
\end{tabular}

Note: different letters indicate the significant differences between different groups (columns). The lowercase letters show significant differences $(\mathrm{P}<0.05)$, while the upper case letters indicate extremely significant differences $(\mathrm{p}<0.01)$; similarly hereinafter.

Table 3. Effects of SACW and its different polar extractions on CAT, GSH-Px activity and GSH contents of rats' liver tissue

\begin{tabular}{lllll}
\hline group & $\mathrm{n}$ & $\mathrm{CAT}(\mathrm{U} / \mathrm{mg}$ prot$)$ & $\mathrm{GSH}(\mathrm{mg} / \mathrm{g}$ prot$)$ & GSH-Px(U/mg prot) \\
\hline blank control group & 8 & $1.92 \pm 0.66$ & $0.055 \pm 0.13$ & $632.24 \pm 59.45$ \\
ether group & 8 & $2.42 \pm 0.37$ & $0.081 \pm 0.14^{\mathbf{a}}$ & $892.11 \pm 43.38^{\mathbf{a}}$ \\
ethyl acetate group & 8 & $2.97 \pm 0.70^{\mathbf{a}}$ & $0.086 \pm 0.09^{\mathbf{a}}$ & $883.37 \pm 56.16^{\mathbf{A}}$ \\
water extract group & 8 & $2.02 \pm 0.56^{\mathbf{c}}$ & $0.072 \pm 0.21^{\text {ac }}$ & $737.79 \pm 72.65^{\text {abc }}$ \\
SACW group & 8 & $3.43 \pm 1.26^{\text {abca }}$ & $0.098 \pm 0.35^{\text {Abca }}$ & $1066.45 \pm 62.04^{\text {Abcv }}$ \\
\hline
\end{tabular}

\subsection{Total flavonoid content of SACW and its different polar extractions}

It can be seen from Table 4, different polar extracts have different total polyphenol and flavonoid contents. The order from high to low contents is as following: SACW group, water extract group, ether group, ethyl acetate group. In all extracts except for the wine group, the content of total flavonoid is the highest in water extract group $(0.157 \mathrm{mg} \mathrm{mL}-1)$, and lowest in the ethyl acetate group (0.059mg mL-1).

Table 4 Total flavonoid contents of different SACW extractions

\begin{tabular}{ll}
\hline Group & Total flavonoid content $/ \mathrm{mg} \cdot \mathrm{mL}-1$ \\
\hline ether group & $0.063 \pm 0.007$ \\
ethyl acetate group & $0.059 \pm 0.012$ \\
water extract group & $0.157 \pm 0.008^{\mathrm{ab}}$ \\
SACW group & $0.283 \pm 0.027^{\mathrm{ABC}}$ \\
\hline
\end{tabular}

\section{Analysis and Discussion}

\subsection{Effects of semen astragali complanati wine and its different polar extracts on body weight and visceral organs of mice}

At the thirtieth day of the experiment, the average weight increases of rats in the semen astragali complanati wine group, water extract group, ethyl acetate group and ether group are $11.65 \%, 14.48 \%$, $23.20 \%$ and $26.20 \%$. Compared with rats in the control group (28.33\%), the weight increases of semen astragali complanati wine group and water extract group reduces significantly. It can be seen that the different extracts of semen astragali complanati have inhibitory effects on the weight growth of mice. Although polyphenol is a natural substance with strong reducing property, and has advantages of high antioxidant activity and low toxicity, phenolic compounds are prototypical toxicants which can solidify proteins and have certain anti nutritional effects on individuals. However, these extracts do not produce significant effects on visceral indexes of mice, indicating no damage to organs of mice. Therefore, it can be concluded that in the range of experimental concentration, semen 
astragali complanati wine and its extracts are almost non-toxic, and the anti nutritional effects do not hamper their antioxidant protection to hepatocytes.

\subsection{The relationship between the flavonoid content of semen astragali complanati and its different polar extracts and the in vivo antioxidant activity of rats}

In this experiment, rats are exposed to extracts of semen astragali complanati wine for 30 days. The polyphenol in wine could interact with hepatocytes for a certain period of time. Flavonoid could enter cells through cell membrane, and preserve in cells in a special form. If cells are subjected to oxidative stress, a large number of harmful free radicals will be produced. Flavonoid can exert their functions of free radicals scavenging to clear excess free radicals, and inhibit lipid peroxidation induced by harmful free radicals, thus protecting the integrity of cell membrane.

The activities of CAT, T-AOC, SOD and GSH-Px and the contents of -SH and MDA in the liver antioxidant enzyme system are main indicators which reflect the metabolism of free radicals, and also indirectly reflect the capacity of liver on scavenging free radical. Flavonoid is strong in vitro antioxidants because of its capacity of scavenging free radicals and acquiring metal. It has been reported in the literature that positively or linear relationship exists between the total phenol and flavonoid contents and the in vitro free radical scavenging ability [10-13].

Flavonoid compounds are found in all extracts of semen astragali complanati wine, and they all show good antioxidant capacities, reducing abilities and scavenging free radical capacities in liver, indicating that flavonoid compounds play an important role in the antioxidant capacity of semen astragali complanati wine. As for different extracts, the total flavonoid content is highest in the water extract group $(0.157 \mathrm{mg} \cdot \mathrm{ml}-1)$, and lowest in ethyl acetate group $(0.059 \mathrm{mg} \cdot \mathrm{ml}-1)$, which shows that flavonoid in astragali complanati wine is mainly composed of polarity materials. Due to the differences between the micro environment of bio film in which the antioxidant and substrate exist and the environment of in vitro experiments, the antioxidant activities are greatly different from in vivo and vitro environments. Fat soluble ethyl ether and ethyl acetate extract groups contain less flavonoid than the water extract group, but their anti oxidation abilities of liver are stronger than the water extract group. The reason may be related to the different antioxidant mechanisms of different kinds of flavonoid extracts.

\subsection{In vivo synergistic antioxidant effects of polyphenol in semen astragali complanati wine and its different polarity extracts}

Experimental results show that all extracts of semen astragali complanati wine have antioxidant effects, and the antioxidant activity of liver of rats from the semen astragali complanati wine group is significantly higher than other groups. It indicates the synergistic effect between different extracts. These extracts cooperate with each other and improve the antioxidant effects of liver, and take the effect of liver protection.

\section{Acknowledgements}

Fund Project: This paper is supported by the National Natural Science Foundation of China [No.31101324] and Funds for Key Research and Development Programs of Shaanxi Province [No.2017NY-167]. It is also supported by the Basal Research Fund for Central Universities of China [No. GK201404006], the Foundation for Technology Transfer Promotion Projects of Xi'an, Shaanxi Province [No. SXSF003] and the Basic Research Funds for Central Universities [No. GK201602005].

\section{References}

[1] D.Q. Wei, Pharmacological action of semen astragali complanati and its extracts, J. Northwest Pharmaceutical Journal. 1 (1986).

[2] C.Y. Liu, Z.L. Gu, R. Han, The protection effects of semen astragali complanati extracts on liver injury of carbon tetrachloride, J. Chinese Traditional and Herbal Drugs. 33 (2002). 
[3] C.Y. Liu, Z.L. Gu, R. Han, L.J. Zhu, W.X. Zhou, C.Y. Guo, the protective effect of flavonoids of semen astragali complanati on $\mathrm{CCl} 4$ and D- galactosamine induced acute liver injury, J. Chinese Traditional and Herbal Drugs. 36 (2005).

[4] C.Y. Liu, Z.L. Gu, K.P. Zhang, W.X. Zhou, C.Y. Guo, the effects of flavonoids of semen astragali complanati on the formation of DMN induced rat hepatic fibrosis, J. Chinese Pharmacological Bulletin. 20 (2004).

[5] M.H. Chen, Study on the chemical composition of Chinese herbal medicine semen astragali complanati, J. Bulletin of Chinese Materia Medica. 12 (1987).

[6] M.H. Chen, Study on the chemical constituents of semen astragali complanati oil, J. China Journal of Chinese Materia Medica.15 (1990).

[7] Q.A. Zhang, X.H. Fan, M. Liu, Z.Q. Zhang, Optimal extraction of flavonoid compounds from semen astragali complanati through response surface method and its ability of scavenging DPPH free radical, J. Natural Product Research and Development. 25 (2013). 